\title{
Validation of a New Animal Model of Vulnerable Plaques by Intravascular Optical Coherence Tomography In Vivo
}

\author{
Yan Fang, ${ }^{1}$ Sining Hu, ${ }^{1}$ Jingbo Hou, ${ }^{1}$ Lingbo Meng, ${ }^{1}$ Shaosong Zhang, ${ }^{2}$ and Bo Yu${ }^{1}$ \\ ${ }^{1}$ Department of Cardiology, The Second Affiliated Hospital of Harbin Medical University, The Key Laboratory of Myocardial Ischemia, \\ Chinese Ministry of Education, Harbin 150086, China \\ ${ }^{2}$ LightLab Imaging Inc., St. Jude Medical, Westford, MA 01886, USA
}

Correspondence should be addressed to Bo Yu, yubodr@163.com

Received 21 July 2012; Accepted 31 August 2012

Academic Editor: M. Ilyas Kamboh

Copyright ( $) 2012$ Yan Fang et al. This is an open access article distributed under the Creative Commons Attribution License, which permits unrestricted use, distribution, and reproduction in any medium, provided the original work is properly cited.

\begin{abstract}
We aimed to establish a rabbit model of vulnerable plaques (VPs) with the morphology and component characteristics of human VPs and to evaluate the microstructural features of VPs in vivo using intravascular optical coherence tomography (OCT). Twelve rabbits underwent endothelial denudation of the carotid artery and consumed a $1 \%$ high-cholesterol diet (HCD). They were equally divided into two groups: group A (modified needle injury) and group B (balloon injury). OCT was undertaken thrice before injury as well as $1 \mathrm{~h}$ and 12 weeks after injury. The degree of acute artery injury after endothelial denudation was detected by OCT. Twelve weeks after injury, OCT showed that both groups generated VPs which had thin fibrous caps and a large lipid core, whereas plaques in group A had smaller lipid arcs $(P<0.0001)$. Histological findings demonstrated that a larger eccentricity index (EI) $(P<0.05)$ and greater infiltration of macrophages $(P<0.05)$ in group A than in group B. Qualitative and morphometric analyses of plaques showed a significant correlation between histological and OCT measurements. A combination of modified endothelial denudation and an HCD in rabbits produced more eccentric lesions similar to those seen in humans. These data suggest that OCT could be a useful tool for evaluation of the degree of injury and VPs in vivo.
\end{abstract}

\section{Introduction}

Rupture of an atherosclerotic plaque is the primary underlying cause of most acute coronary events and strokes [1, 2]. A "vulnerable plaque" (VP) is considered to be prone to rupture. It is characterized as a large pool of lipids with an overlying thin fibrous cap that is heavily infiltrated by macrophages $[3,4]$. Besides plaque components, morphological characteristics such as lesion eccentricity also provide important prognostic information for vulnerable lesions. Compared with concentric plaques, eccentric plaques are usually associated with not only cerebrovascular and cardiovascular symptoms, but also the progression of atherosclerotic disease [5-7].

To predict VP rupture, methods such as intravascular ultrasound (IVUS) and magnetic resonance imaging (MRI) have been used to identify directly VP features in vivo in some experimental studies $[8,9]$. However, the lower resolution of these modalities has restricted their application for evaluation of the microstructural features of atherosclerotic plaques. Optical coherence tomography (OCT) is a novel intravascular imaging modality that uses near-infrared light with high image resolution $(\approx 10 \mu \mathrm{m})$. OCT allows for unparalleled imaging of the plaque composition [10], such as fibrous-cap thickness (FCT) and lipid content. It has been considered to be one of the most promising tools to detect the key features of plaque rupture and to assess the vascular response to stenting $[11,12]$.

The further study of the development of VPs and intraarterial treatment of stabilizing plaques is important, and an appropriate animal model is required. The aim of the present study was to establish an animal model with morphological and structural characteristics similar to those seen in human VPs. We also wished to evaluate the reliability of OCT technology for repeated observation of lesion features in vivo.

\section{Methods}

2.1. Ethical Approval of the Study Protocol. The study protocol was approved by the Ethics Committee of the Second 
Affiliated Hospital of Harbin Medical University (Harbin, China).

2.2. Experimental Protocol. Sixteen adult male New Zealand white rabbits $(\approx 3.0 \mathrm{~kg})$ were housed continuously at the animal care facilities of the Second Affiliated Hospital of Harbin Medical University. Sixteen rabbits were fed a $1 \%$ high-cholesterol diet (HCD) for 2 weeks before and 12 weeks after endothelium denudation. They were divided into two groups according to the pattern of vessel injury. In group A $(n=8)$, eccentric endothelial denudation was induced by an inflexible modified metal needle which had a blunt and rounded front head (diameter, 0.0285 inch); a partial and longitudinal injury to the vascular wall was generated (Figure 1). For rabbits in group B $(n=8)$, concentric endothelial denudation was achieved using a balloon. Three uninjured rabbits were fed a normal chow diet. They were used as controls to compare the normal histological and OCT appearance of the vascular wall to that of diseased rabbits.

2.3. Injury Using Balloons and Needles and Imaging by OCT. After 2 weeks of the $1 \%$ HCD diet, rabbits were anesthetized with ketamine $(5 \mathrm{mg} / \mathrm{kg})$, xylazine $(5 \mathrm{mg} / \mathrm{kg})$, and acepromazine $(0.75 \mathrm{mg} / \mathrm{kg})$ given via the intramuscular route. Anesthesia was maintained during the procedure with isoflurane inhalation via a mask [13]. Rabbits were heparinized and a median incision in the neck region made under local anesthesia induced by $1 \%$ lidocaine hydrochloride. After the right common carotid artery was exposed surgically, a 1-2 mm arteriotomy was made at the external carotid artery. An OCT wire was inserted through the cut into the common carotid artery. OCT was obtained using a time-domain OCT System (Light Lab Imaging, Westford, MA, USA) with an image wire (crossing profile, 0.014 inches; Light Lab Imaging) at a pullback speed of $3 \mathrm{~mm} / \mathrm{s}$ during intermittent flushing with $0.9 \%$ (physiological) saline through the guiding catheter to displace blood transiently.

After withdrawing the OCT image wire, a 3-F Fogarty catheter was introduced through the same cut-down in the rabbits of group B. The balloon was then inflated with $0.3 \mathrm{~mL}$ saline, and the catheter gently retracted to the external carotid artery as described previously [13]. In group-A rabbits, the modified metal needle replaced the balloon and was used to make a partial and longitudinal endothelial denudation. Vessel injury was completed thrice in each rabbit of groups A and B. The balloon or needle was then removed. One hour later, the OCT image wire was introduced through the same cut-down again. With the saline flush, serial OCT images were obtained. After pulling out the OCT image wire, the external carotid branch was ligated. Postoperative therapy was aspirin $(100 \mathrm{mg}$, p.o.) and ampicillin $(250 \mathrm{mg}$, i.m.) for the next 3 days.

Twelve weeks later, OCT was repeated immediately on the same carotid artery before killing. OCT was undertaken thrice before injury as well as $1 \mathrm{~h}$ and 12 weeks after injury. The procedures detailed above were undertaken in the right common carotid artery of each rabbit (Figure 1).
2.4. Histopathology and Immunohistochemistry. After killing, each carotid artery underwent perfusion fixation and was harvested. Each carotid artery was cut into three segments (proximal, middle, distal). The proximal end of each segment was marked with a suture ligature on its anterior side to avoid confusion.

Samples were embedded in paraffin and cut into serial transverse sections of $5 \mu \mathrm{m}$ for histopathological and immunohistochemical analyses. Hematoxylin and eosin (H\&E) and Masson's trichrome staining were carried out to show structural changes in vascular walls and to detail the plaque components [14]. If atherosclerotic lesions were identified by morphological analyses, additional immunohistochemical staining of macrophages and smooth muscle cells (SMCs) was carried out as described by Tian et al. [15]. Staining with Picrosirius red was also done to identify the type of collagen fibers within the fibrous caps of plaques under polarized light (type I, orange; type III, green).

2.5. Plasma Lipid Analyses. Blood samples were collected at baseline and before killing. Plasma levels of total cholesterol (TC) and triglycerides (TG) were detected using standard enzymatic techniques. Levels of high-density lipoprotein-cholesterol (HDL-C) and low-density lipoproteincholesterol (LDL-C) were also measured.

2.6. OCT Analyses. Normal OCT and the corresponding histology cross-sections of carotid arteries were acquired from uninjured and normal-diet rabbits. OCT images from HCD rabbits before endothelial injury were used as the baseline. Acute vessel injury was evaluated by OCT after balloon and needle injury. According to previous histology results [16], mild injury was defined as endothelial denudation with or without punctated breaks in the internal elastic lamina (IEL) and without medial laceration. Severe injury was defined as disruption of the media and even large lacerations of media extending through the adventitia.

OCT images before killing were used to assess the characteristics of atherosclerotic lesions. Plaque type as well as the length of the plaque and lipid core were determined by OCT as described previously $[10,15,17]$. FCT was measured at five locations of the fibrous cap on each cross-sectional image and the average value calculated. The minimum FCT was measured at its thinnest part. Measurements of the mean FCT, the mean area of the lumen, and the mean value of the arc of the lipid core were calculated at 1 cross-sectional image per $1 \mathrm{~mm}$ interval of lesion. The percentage of luminal stenosis was calculated by comparing the mean lumen area of the lesion to that of a reference site using the following formula:

$$
\% \text { stenosis }=\left[1-\left(\frac{\text { mean lumen area }}{\text { lesion }}\right) \times 100\right] .
$$

Within $10 \mathrm{~mm}$ of the distal and proximal end of the plaque, the approximate normal vessel wall was regarded to be the reference site. The reference lumen area was measured as the mean value between the distal and proximal reference lumen area. Finally, the presence of thrombus and plaque fissure was 

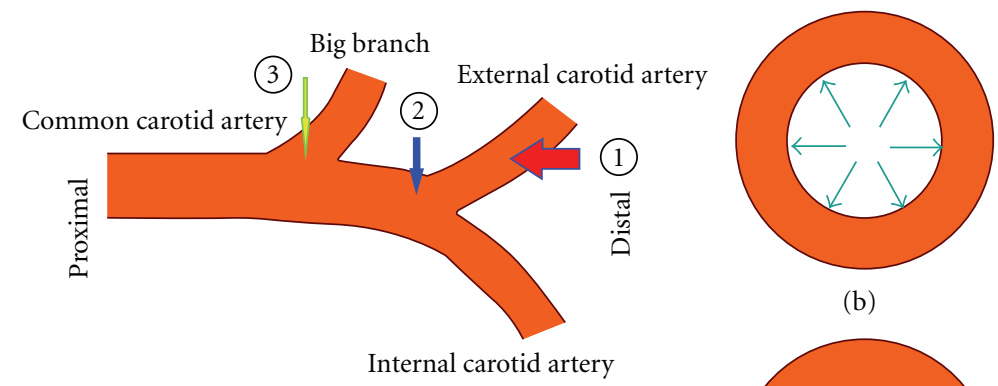

(b)

(a)

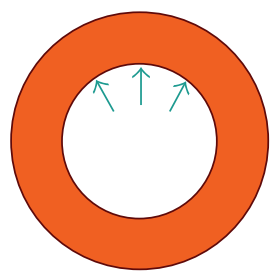

(d)

(c)

FIGURE 1: Procedure of OCT imaging and injury (schematic). (a) The imaging catheter and injury-causing device are advanced initially to the common carotid artery through the external carotid branch (arrow (1)). At the end of the study, the imaging catheter is again advanced to the common carotid artery through the region between the old incision and the big branch (arrow (2) or arrow (3)). (b) Balloon injury results in endothelial denudation with the formation of concentric lesions (arrows indicate the sites of endothelial denudation). (c) A modified metal needle generates an endothelial injury with the formation of eccentric lesions (arrows indicate the sites of endothelial denudation). (d) A modified metal needle with a blunt and rounded front head and diameter of 0.0285 inches was employed.

also noted. OCT images were analyzed by two independent observers who were blinded to the procedural characteristics.

\subsection{Histological Analyses and Matching of Histological Sections} and OCT Images. The right carotid artery bifurcation and its big branch were used as the internal anatomic markers for localization to match histological and OCT cross-sectional images as well as to match OCT images at different time points. Atherosclerotic plaques identified at histology were classified according to criteria set by the American Heart Association (AHA) [18]. The area and thickness of crosssectional media and plaques were derived from the inner sides of the outlines of the lumen and the adventitia. The eccentricity index (EI) was calculated as the ratio between the minimal and maximal thickness of media and plaque (MPT) at the cross-sectional image of maximal plaque formation. The plaque was defined as an eccentric plaque if the EI was $\geq 0.5$ and as a concentric plaque if $<0.5$ [5]. FCT and lumen area were also measured. Furthermore, the area percentage of macrophages foam cells and SMCs was quantified (at $\times 100$ magnification) within digitized immunohistochemically stained plaques. The measured region of interest included the maximum amount of macrophages and an overlying fibrous cap. All slides were analyzed by computerized planimetry using Image-Pro Plus 4.5 (Media Cybernetics, Silver Spring, MD, USA).

2.8. Statistical Analyses. The values for histology and OCT measurements were expressed as mean \pm standard deviation. The Student's $t$-test was done to evaluate the level of significance for a given measurement between the two groups. The intraclass correlation coefficient (ICC) was calculated to evaluate the agreement between OCT and histological findings. Statistical analyses were done using SAS ver9.1.3 (SAS Institute, Cary, NC, USA). $P<0.05$ (two-sided) was considered to significant.

\section{Results}

Of 4 rabbits that did not complete the experiment protocol, 2 from group A died prematurely from anesthesia accidents, and 2 from group B had chronic total occlusion (CTO) in the distal right carotid artery identified by histological analyses. CTO lesions comprised mainly macrophage foam cells and extracellular lipids (Figure 3(F)). CTO lesions had no corresponding OCT images. Each of the remaining 12 rabbits underwent further OCT and histological examination.

3.1. Changes in Plasma Levels of Lipids. The baseline levels of TC, TG, HDL-C, and LDL-C were $2.46 \pm 1.51,1.17 \pm$ $0.37,0.87 \pm 0.33$, and $0.66 \pm 0.45 \mathrm{mmoL} / \mathrm{L}$, respectively. The levels of TC, TG, HDL-C, and LDL-C increased to $37.86 \pm$ $1.93,16.71 \pm 4.46,10.53 \pm 3.04$, and $28.08 \pm 5.26 \mathrm{mmoL} / \mathrm{L}$, respectively (all $P<0.001$ ). However, a significant difference was not observed in any of these measured plasma lipid levels between the rabbits in group A and group B (Supplementary Table, available at doi:10.1155/2012/469726).

3.2. Acute Vessel Injury Evaluated by OCT. Mild injury and severe injury were found in both injury groups (Figure 2). Further statistical comparisons between the two injury patterns were not carried out because an accurate number of total injured cross-sections was not available. Mild injury without punctated breaks in the IEL was not detected by OCT. 


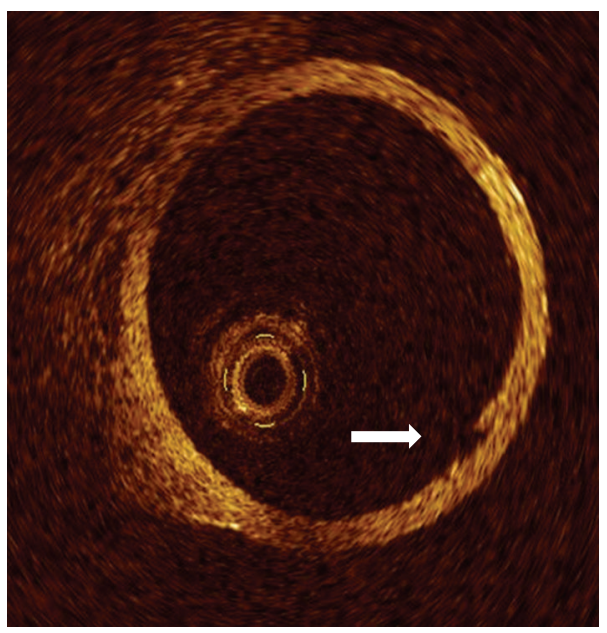

(a)

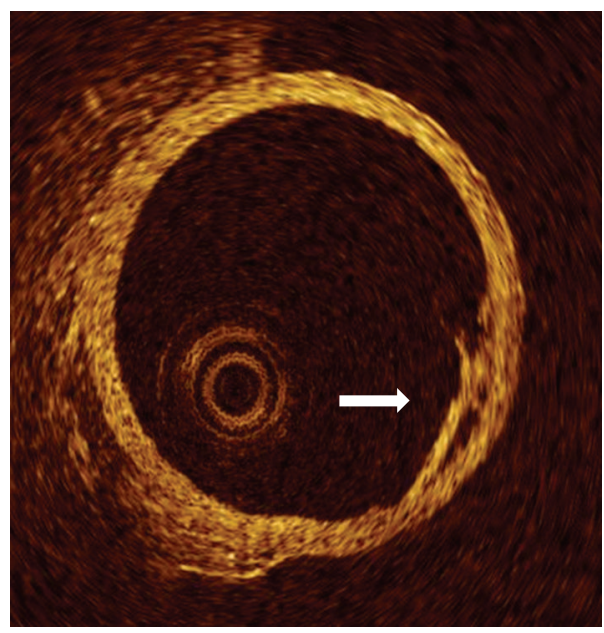

(b)

FIGURE 2: OCT images of acute vessel injury. (a) Mild vessel injury with punctated breaks in the IEL (arrow). (b) Severe vessel injury with disruption of the media (arrow).

3.3. OCT Findings of Atherosclerotic Lesions and the Corresponding Histopathological Features. Atherosclerotic lesions were not observed in the vessels of control rabbits. Tissue sections from control rabbits showed layering of normal carotid arteries (Figures 3(A1)-3(A4)). The media contained abundant collagen (Figure 3(A2)), SMCs (Figure 3(A3)), and few macrophages (Figure 3(A4)) and they showed high backscattering intensity on OCT images (Figure 3(A1)). The adventitia (which consists primarily of loose connective tissue) exhibited the low backscattering intensity. The media and adventitia were clearly identified by OCT. The intima could not be detected because its thickness was $<10 \mu \mathrm{m}$.

In the experimental groups, 12 nonocclusive carotid arteries from 12 rabbits were analyzed by OCT. The OCT appearance of one lesion (Figure 3(B1)) showed highly reflective subintimal areas. These were documented as typeIII lesions and comprised SMCs (Figure 3(B3)) and small extracellular accumulations of lipids (Figure 3(B4)) at histology [18]. A total of 23 lesions in 12 carotid arteries with a fibrous cap and large extracellular lipid cores were documented as advanced plaques ( $\geq$ type-IV lesion) according to criteria noted previously [19]. OCT images of type-IV lesions (Figure 3(C1)) were characterized by signal-poor regions (lipid pools, Figure 3(C3)) with an overlying thin fibrous cap (Figure 3(C3)) which consisted mainly of collagen fibers (type I, orange; type III, green; Figure $3(\mathrm{C} 4)$ ). The OCT appearance of type- $\mathrm{V}$ plaques was similar to that of typeIV lesions but with a thickened fibrous cap (Figure 3(D4)) overlaying a lipid core. The OCT and corresponding histological images of type-VI plaques (Figures 3(E1)-3(E4)) showed a visible adherent white thrombus attached to a lipidrich fibrous plaque. Additionally, neovessels, cholesterol crystals, and intraplaque hemorrhage were observed by histopathological examination in the atherosclerotic lesions of the experimental groups (Figures $3(\mathrm{G})-3(\mathrm{I})$ ). Cholesterol crystals could not be detected by OCT because of the barrier of lipids and thick caps.
3.4. Comparison of Total Plaque Characteristics between the Two Experimental Groups by OCT. Twenty-four advanced lesions were identified by OCT. Twenty-three lesions were confirmed as being "advanced lesions" by histology [18]. The other lesion was a type-III lesion. Overall, 261 OCT cross-sections of atherosclerotic lesions were analyzed. OCT was used to correctly diagnose 255/261 (97.7\%) as advanced lesions and misdiagnosed 6/261 (2.3\%) type-III crosssections as type-IV lesions.

The OCT features of advanced lesions in group A $(n=$ $12)$ and group $\mathrm{B}(n=11)$ were collected and compared in vivo (Table 1). Significant differences between group A and group B were not observed with respect to the average and minimum FCT, lipid-core length, plaque length, average lumen area, and the average stenosis ratio. However, the balloon injury group had a larger lipid arc.

3.5. Comparison of the Histological Findings between Plaques with Maximum Formation from the Two Injury Groups. Atherosclerotic plaques with maximum formation were selected from each right carotid artery of rabbits in the two groups (Figure 4). Twelve plaques were selected and the microstructures between the two groups were compared (Table 2). Although the two injury patterns could initiate eccentric lipid-rich plaques, those from the needle-injury group exhibited a higher EI, thinner FCT, and greater infiltration of inflammatory cells. Plaques from the ballooninjury group had greater lipid content in the mean plaque area and mean plaque thickness (mean, minimum, and maximum), but there was no significant difference in minimum FCT and lumen area.

3.6. Agreement between OCT and Histopathology Images. Thirteen representative OCT and corresponding histology cross-sections were selected from 23 advanced lesions to ascertain the agreement between OCT and histopathology. 


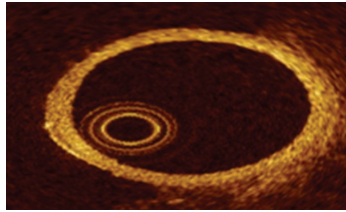

(A1)

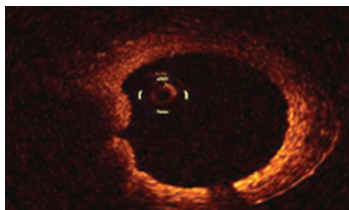

(B1)

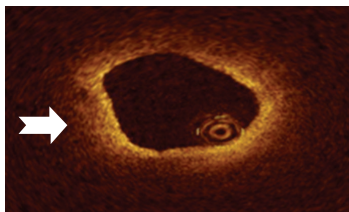

(C1)

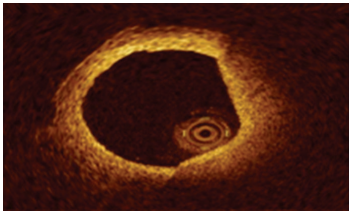

(D1)

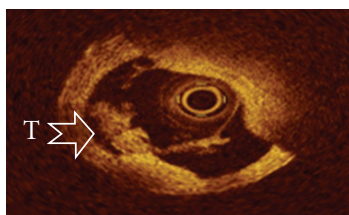

(E1)

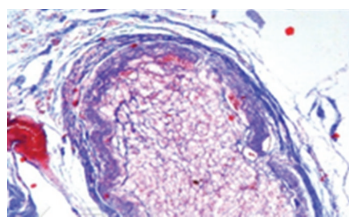

(F)

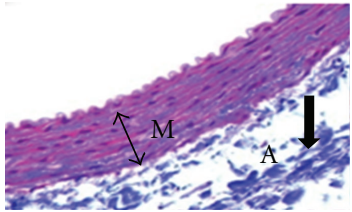

(A2)

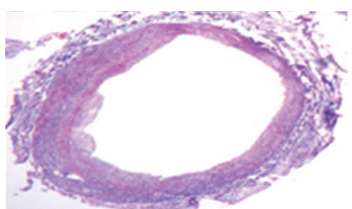

(B2)

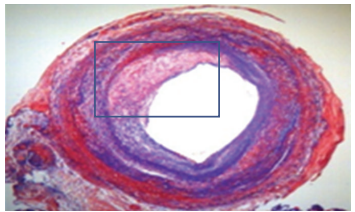

(C2)

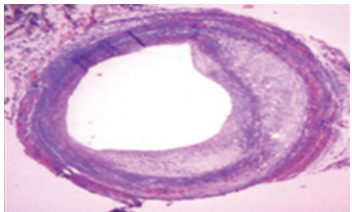

(D2)

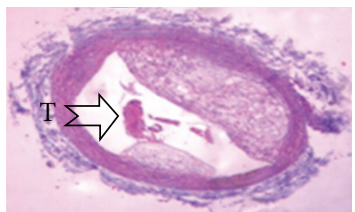

(E2)

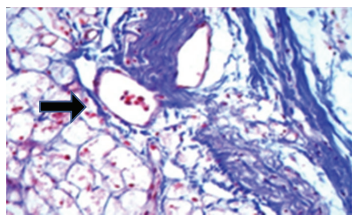

(G)

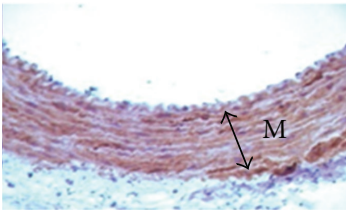

(A3)

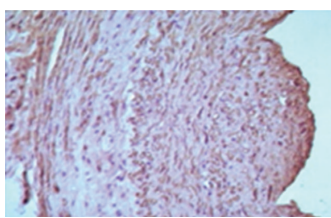

(B3)

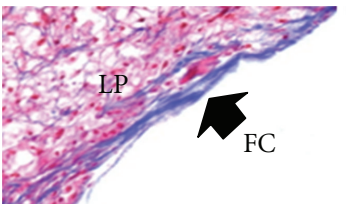

(C3)

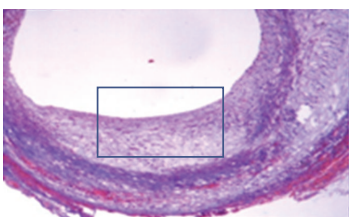

(D3)

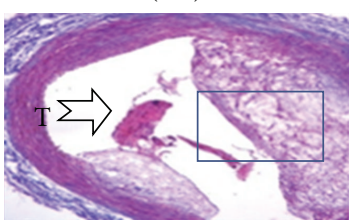

(E3)

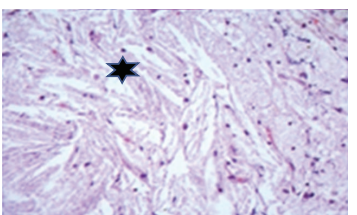

(H)

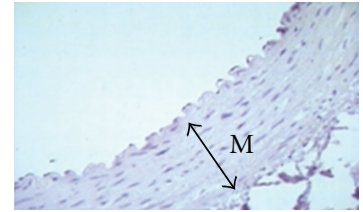

(A4)

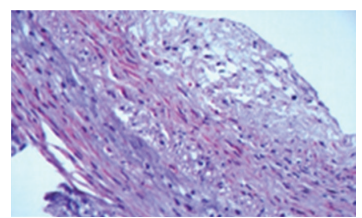

(B4)

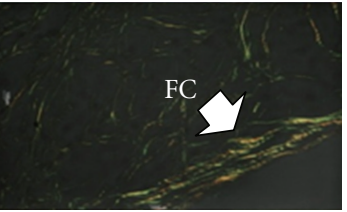

(C4)

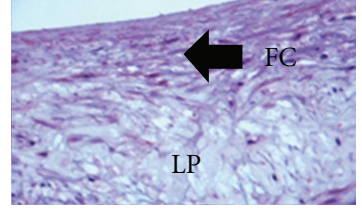

(D4)

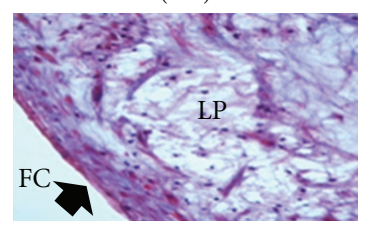

(E4)

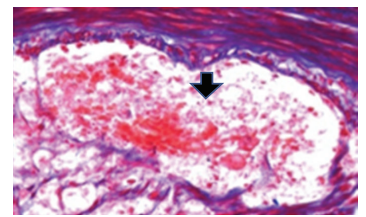

(I)

FIGURE 3: Histological features of normal and atherosclerotic lesions in rabbits and the corresponding OCT images. (A1)-(A4): OCT and histology images of a normal vessel; (B1)-(B4): type-III plaque; (C1)-(C4): type-IV plaque. D1-D4: type-V plaque; (E1)-(E4): type-VI plaque; (F): lesion showing chronic total occlusion (CTO). The lumen is full of foam cells and lipid components; (G): medial neovessels in lesions with CTO. The neovessels (arrow) run longitudinally and contain red blood cells (RBCs). (H): A cholesterol crystal in a typeVa plaque. The cholesterol crystal looks like a spindle and accumulates into one part of the necrotic core; (I): intraplaque hemorrhage. RBCs have accumulated in the lipid pool. Staining is by Masson's trichrome (A2, B2, B4, C2, C3, D2-D4, E2-E4, and F-I), smooth muscle cell $\alpha$-actin immunohistochemisty (A3 and B3), macrophage immunohistochemical identification (A4), and Picrosirius Red viewed under polarized light (C4). Original magnification of B2, C2, D2, and E2 is $\times 40$; (A2)-(A4), (D3), (E3), and (F) is $\times 100$. (B3), (B4), (C3), (C4), (D4), (E4), and (G)-(I) is $\times 400$. M denotes the media; A, adventitia; FC, fibrous cap; LP, lipid pool; T, thrombus.

Compared with histopathology, OCT measurement of the mean plaque area showed an acceptable correlation, whereas measurements of FCT and the lipid arc by OCT were extremely accurate (Table 3 ). Because of the shrinkage of the lumen of tissue sections, OCT measurements of lumen area were significantly different compared with the corresponding histological examination (Table 3 ). In addition, mean plaque thickness and the EI could not be detected by OCT due to its limited penetrating power of lipid content.

\section{Discussion}

In the present study, we modified the pattern of denudation of endothelial cells and developed a more common model of eccentric VPs that reproduced the features of human VPs. In the development of atherosclerotic diseases, balloon injury and hyperlipidemia are thought to cause injury to endothelial cells and result in further atherosclerotic lesions $[20,21]$. A combination of mechanical damage and lipid 

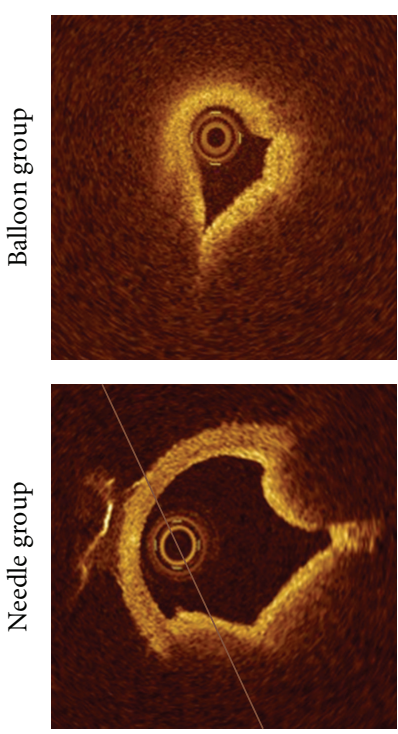

(a) OCT
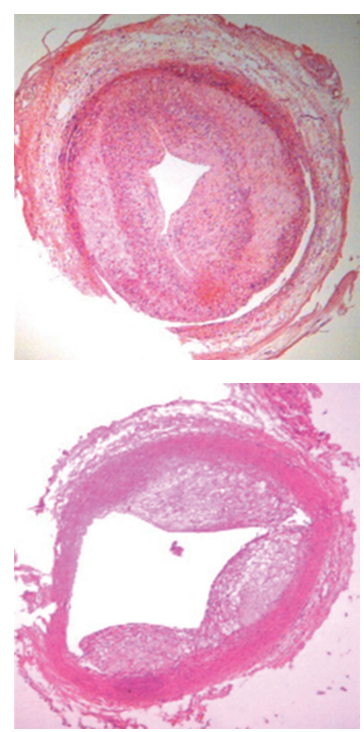

(b) HE
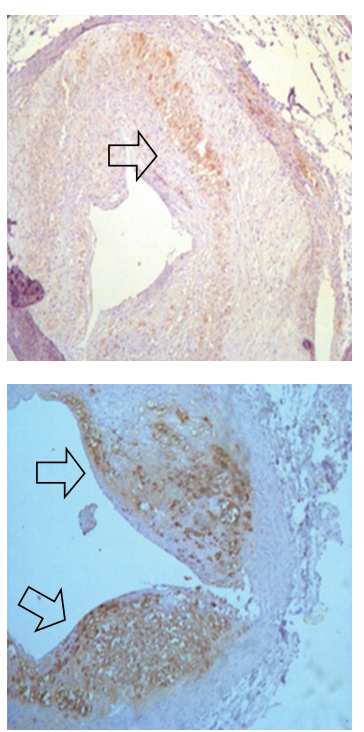

(c) Macrophage
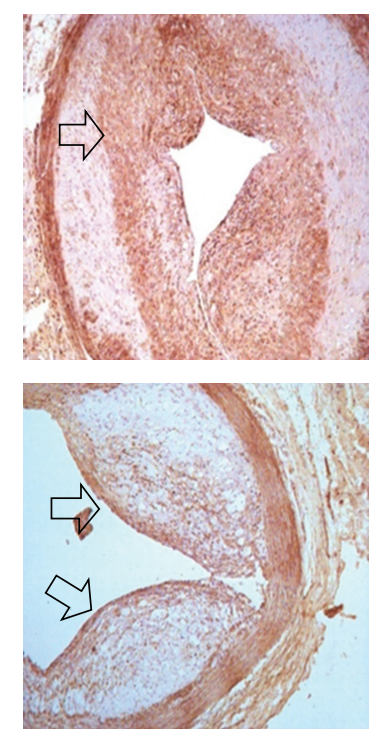

(d) SMC

FIGURE 4: Representative cross-sectional images of the balloon-injury group and needle-injury group. (a): a concentric plaque with a lipidrich core and thick fibrous cap from the balloon-injury group and eccentric plaque with a lipid-rich core and thin fibrous cap from the needle-injury group; (b): corresponding histology sections with a similar shape from the two injury groups; (c): immunostaining for macrophages by anti-CD68 antibody (arrows in (c)), which demonstrate greater accumulation of macrophages in the needle-injury group relative to the balloon-injury group; (d): immunostaining for smooth muscle cells (SMCs) by anti-SMC $\alpha$-actin antibody. The fibrous cap of the plaque in the balloon-injury group contains more SMCs compared with that in the needle-injury group. The original magnification in figure (b) is $\times 40$, whereas in (c) and (d) it is $\times 100$.

TABLE 1: Comparison of total plaque characteristics in the two experimental groups by OCT.

\begin{tabular}{|c|c|c|c|c|}
\hline Feature & Group A $(n=12)$ & Group B $(n=11)$ & $t$ & $P$ \\
\hline Average FCT (mm) & $0.0896 \pm 0.0136$ & $0.0988 \pm 0.0236$ & -1.150 & 0.2630 \\
\hline Minimum FCT (mm) & $0.0483 \pm 0.0136$ & $0.0518 \pm 0.0098$ & -0.978 & 0.3392 \\
\hline Lipid core length (mm) & $9.7667 \pm 5.3181$ & $11.7364 \pm 8.2181$ & -0.688 & 0.4987 \\
\hline Plaque length (mm) & $11.6083 \pm 5.5934$ & $12.8909 \pm 7.9551$ & -0.450 & 0.6570 \\
\hline Average LA $\left(\mathrm{mm}^{2}\right)$ & $1.8203 \pm 0.9462$ & $1.2126 \pm 0.4916$ & 1.960 & 0.0673 \\
\hline Average lipid $\operatorname{arc}\left({ }^{\circ}\right)$ & $149.0058 \pm 27.9580$ & $255.3919 \pm 55.3932$ & -5.740 & $<0.0001$ \\
\hline Average stenosis ratio (\%) & $22.38 \pm 17.64$ & $19.48 \pm 14.00$ & 0.434 & 0.6684 \\
\hline
\end{tabular}

FCT: fibrous cap thickness; LA: lumen area.

TABLE 2: Histological findings of plaques from the two experimental groups.

\begin{tabular}{|c|c|c|c|c|}
\hline Feature & Group A $(n=6)$ & Group B $(n=6)$ & $t$ & $P$ \\
\hline Average FCT $(\mu \mathrm{m})$ & $55.01 \pm 28.67$ & $141.20 \pm 76.57$ & -2.58 & 0.0273 \\
\hline Minimum FCT $(\mu \mathrm{m})$ & $48.20 \pm 25.08$ & $83.80 \pm 39.50$ & -1.864 & 0.0920 \\
\hline Average lipid $\operatorname{arc}\left(^{\circ}\right)$ & $141.67 \pm 69.69$ & $263.33 \pm 82.62$ & -2.757 & 0.0207 \\
\hline CD68 (\%) & $28.74 \pm 14.67$ & $10.69 \pm 7.39$ & 2.692 & 0.0226 \\
\hline SMCs (\%) & $26.08 \pm 12.83$ & $29.52 \pm 11.27$ & -0.493 & 0.6330 \\
\hline $\mathrm{LA}\left(\mathrm{mm}^{2}\right)$ & $0.898 \pm 0.650$ & $0.554 \pm 0.284$ & 1.188 & 0.2624 \\
\hline $\mathrm{MP}$ area $\left(\mathrm{mm}^{2}\right)$ & $1.175 \pm 0.421$ & $2.724 \pm 1.172$ & -3.048 & 0.0213 \\
\hline Min MPT (mm) & $0.098 \pm 0.031$ & $0.396 \pm 0.165$ & -4.351 & 0.0063 \\
\hline Average MPT (mm) & $0.314 \pm 0.069$ & $0.712 \pm 0.258$ & -3.658 & 0.0115 \\
\hline Max MPT (mm) & $0.529 \pm 0.137$ & $1.028 \pm 0.407$ & -2.844 & 0.0288 \\
\hline Average EI & $0.804 \pm 0.077$ & $0.605 \pm 0.134$ & 3.148 & 0.0104 \\
\hline
\end{tabular}

FCT: fibrous cap thickness; LA: lumen area. MPT: media and plaque thickness; MPA: media and plaque area; EI: eccentric index; SMCs: smooth muscle cells. 
TABLE 3: Agreement between OCT and histological findings.

\begin{tabular}{|c|c|c|c|c|}
\hline Feature & OCT $(n=13)$ & Histology $(n=13)$ & $\mathrm{ICC}^{*}(95 \% \mathrm{CI})$ & $P^{\#}$ \\
\hline Average FCT (mm) & $0.0549 \pm 0.0268$ & $0.0673 \pm 0.0453$ & $0.656(0.22-0.879)$ & 0.0034 \\
\hline Average lipid $\operatorname{arc}\left({ }^{\circ}\right)$ & $160.56 \pm 71.43$ & $181.09 \pm 83.82$ & $0.882(0.61-0.964)$ & $<0.0001$ \\
\hline $\mathrm{LA}\left(\mathrm{mm}^{2}\right)$ & $1.9608 \pm 0.3297$ & $0.7331 \pm 0.2765$ & $0.04(-0.029$ to 0.226$)$ & 0.2525 \\
\hline
\end{tabular}

* The intraclass correlation coefficient (ICC) was used to evaluate the agreement between OCT and histological findings.

${ }^{\#} P<0.05$ indicates the agreement between OCT and histological findings.

CI: confidence interval; FCT: fibrous cap thickness; LA: lumen area.

toxicity has been shown to accelerate atherogenesis and generate types of atherosclerotic plaques within 8-12 weeks in rabbits $[14,15,22]$. Balloon injury might cause concentric denudation of endothelial cells lining the luminal site of vessel walls. After endothelial injury, a series of pathologic processes lead to the development of atherosclerotic plaques at injury sites [23]. Our results showed that balloon injury could generate eccentric plaques, but their EI was low and the morphology of plaques was concentric (or approx. concentric). We hypothesized that local endothelial denudation could generate eccentric atherosclerotic lesions. Hence, we chose a modified needle which had a blunt and rounded front head to cause partial and longitudinal injury to vascular walls. After 12 weeks of an HCD, histology results and OCT showed that the needle-injury group could generate more eccentric plaques which had a similar morphology to that of human VPs compared with the balloon-injury group.

In humans, a thin-capped fibroatheroma (TCFA) is defined as a plaque with a thin fibrous cap (thinnest part, $\leq 65 \mu \mathrm{m}$ ) overlying a large lipid pool ( $\geq 2$ quadrants) [24]. This critical threshold of $65 \mu \mathrm{m}$ has been derived from the fragments of atherosclerotic plaques observed in postmortem examinations. Our histological findings showed similar results. The thinnest FCT derived from the fragments of plaques in the needle-injury group $(48.20 \pm 25.08 \mu \mathrm{m})$ was close to the values noted in humans $(65 \mu \mathrm{m})$ and rabbits $(57 \pm 15 \mu \mathrm{m})$ [14]. In addition, the OCT-detected average FCT in the two groups was close to the critical FCT of rupture-prone coronary plaques in humans $(<80 \mu \mathrm{m}$ for the thinnest FCT and $<188 \mu \mathrm{m}$ for the most representative FCT) [25]. Furthermore, those plaques had a large lipid arc of $\geq 2$ quadrants. Hence, the plaques of the needle-injury group were equipped with the representative features of human rupture-prone plaques.

In all 12 rabbits, we observed advanced atheromatous lesions by OCT and histological examination. Human autopsy studies have shown that advanced lesions (types IV and Va) are especially prone to disruption [19]. Those tears may occur more frequently in regions of lesions with greater infiltration of macrophage foam cells [26]. Indeed, local inflammation can induce the expression of collagenase and inhibit the expression of proteolytic inhibitors, thereby rendering the fibrous cap weak and susceptible to rupture $[23,27]$. By contrast, vascular SMCs may be important in maintaining plaque stability through the formation of a firm fibrous cap. In the present study, there was similar SMC content within plaques in the two groups. However, plaques in the needle-injury group had more macrophage foam cells compared with those of the balloon-injury group. The greater infiltration of inflammatory cells might increase the vulnerability of plaques. In addition, the neovascularization of plaques and intraplaque hemorrhage also increase the vulnerability of plaques $[28,29]$. In the present study, the fibrous cap of plaques with intraplaque hemorrhage was fissured in the shoulder region. Hence, erythrocytes might intrude from the lumen into the plaque, resulting in intraplaque hemorrhage.

The needle-injury group could generate more eccentric, lipid-rich plaques whose caps were thin, fibrous and infiltrated with macrophages. They mimicked human VPs in terms of morphology and plaque composition.

The present study showed that OCT was a useful tool to identify the degree of injury immediately after endothelial denudation and for evaluation of the prognosis. Previous histological examination indicated that severe injury to the vascular wall resulted in a larger neointimal lesion compared with a mild degree of injury [16]. It is possible that removal of the medial barrier facilitated the intimal migration of adventitial cells [30]. Previous results were identified according to the histological examination or autopsy and could not be used to detect immediate vascular injury in situ of the same vessel due to the limitation of intravascular imaging facilities.

OCT could be used to correctly detect advanced lesions and their microstructural features in rabbits. For advanced plaques ( $\geq$ type IV) in humans, intracoronary OCT has been reported to accurately identify lipid pools [10], acute thrombus [13], and FCT [24]. In the present study, OCT provided clear imaging and quantitative analyses of lipid pools and FCT in vivo in rabbits. The correlation observed between histological and OCT measurements may allow evaluation of the effects on FCT and the lipid arc in plaques under different drug treatments without killing the animals.

In the present study, most rabbits underwent final OCT at the distal region of the common carotid artery (arrow (2) of Figure 1). After ligating the distal end of the common carotid artery, the big-branch vessel could guarantee unobstructed blood flow. Hence, it provided the possibility of further imaging which could be undertaken at the site of the big-branch vessel (arrow (3) of Figure 1). The unique anatomic structure of the carotid artery in rabbits permits serial in vivo monitoring of the evolving characteristics of the same lesion over time. Our previous animal study demonstrated the feasibility of OCT to evaluate the same artery twice in vivo [15]. Utilizing this rabbit model, OCT could be used to not only accurately evaluate VP 
features, but also to observe and measure the same plaques more than once. This model could guide the design of invasive imaging approaches for the detection and treatment of VPs. It could also allow implantation of stent materials evaluated by OCT.

One limitation of this rabbit model was the absence of normal human-like layering of the intima and media in the coronary vessel wall in OCT images for normal rabbits. This could be because the carotid artery of rabbits is elastic whereas that of humans is muscular. Besides, OCT shows poor penetration of tissue (1-2 $\mathrm{mm}$ ) and high attenuation in lipid plaques. The binding between the media and adventitia was also unclear in OCT images in this model. OCT could not be used to detect definitively the thickness of the media and neointima. We could not recommend measurement of the thickness of the media and neointima by OCT in this rabbit model. One way to overcome this limitation of penetration is to combine OCT with other modalities capable of imaging through blood. IVUS can be used to detect binding between the media and adventitia, and has been shown to provide additional information that may be related to plaque vulnerability. Another limitation was that the method of triggering plaque rupture was not done before killing. Therefore, further studies are needed to evaluate the appearance between ruptured and nonruptured plaques in vivo in OCT images.

\section{Conclusions}

We developed an animal model of VPs with more eccentric plaques and a similar morphology to that seen in human VPs. OCT is a useful tool to detect the degree of acute injury as well as advanced lesions and their microstructural features in vivo. A combination of OCT and this modified model of VPs could be an important research tool for the understanding and treatment of VPs in acute coronary syndromes.

\section{Acknowledgments}

This study was supported by the National Natural Science Foundation of China (Grant no. 30871064). The authors acknowledge the help of Tao Zhang, Haibo Jia, Shaotao Zhang and Linxing Feng for their assistance in this study. The authors are grateful to the staff of the Department of Cardiology of the Second Affiliated Hospital of Harbin Medical University for the use of the Intervention Operation Room and for their guidance.

\section{References}

[1] M. J. Davies, "Anatomic features in victims of sudden coronary death: coronary artery pathology," Circulation, vol. 85, no. 1, pp. I19-I24, 1992.

[2] A. P. Burke, A. Farb, G. T. Malcom, Y. H. Liang, J. Smialek, and R. Virmani, "Coronary risk factors and plaque morphology in men with coronary disease who died suddenly," The New England Journal of Medicine, vol. 336, no. 18, pp. 1276-1282, 1997.

[3] M. Naghavi, P. Libby, E. Falk et al., "From vulnerable plaque to vulnerable patient: a call for new definitions and risk assessment strategies: part I," Circulation, vol. 108, no. 14, pp. 1664-1672, 2003.

[4] R. Virmani, A. P. Burke, A. Farb, and F. D. Kolodgie, "Pathology of the vulnerable plaque," Journal of the American College of Cardiology, vol. 47, no. 8, pp. C13-C18, 2006.

[5] M. Yamagishi, M. Terashima, K. Awano et al., "Morphology of vulnerable coronary plaque: insights from follow-up of patients examined by intravascular ultrasound before an acute coronary syndrome," Journal of the American College of Cardiology, vol. 35, no. 1, pp. 106-111, 2000.

[6] T. Ohara, K. Toyoda, R. Otsubo et al., "Eccentric stenosis of the carotid artery associated with ipsilateral cerebrovascular events," American Journal of Neuroradiology, vol. 29, no. 6, pp. 1200-1203, 2008.

[7] P. Nair, L. Gruberg, and R. Beyar, "The eccentric lumenology," Acute Cardiac Care, vol. 8, no. 2, pp. 87-94, 2006.

[8] A. Phinikaridou, F. L. Ruberg, K. J. Hallock et al., "In vivo detection of vulnerable atherosclerotic plaque by MRI in a rabbit model," Circulation, vol. 3, no. 3, pp. 323-332, 2010.

[9] G. Chiesa, C. Di Mario, N. Colombo et al., "Development of a lipid-rich, soft plaque in rabbits, monitored by histology and intravascular ultrasound," Atherosclerosis, vol. 156, no. 2, pp. 277-287, 2001.

[10] H. Yabushita, B. E. Bouma, S. L. Houser et al., "Characterization of human atherosclerosis by optical coherence tomography," Circulation, vol. 106, no. 13, pp. 1640-1645, 2002.

[11] I. Ben-Dor, M. Mahmoudi, A. D. Pichard, L. F. Satler, and R. Waksman, "Optical coherence tomography: a new imaging modality for plaque characterization and stent implantation," Journal of Interventional Cardiology, vol. 24, no. 2, pp. 184192, 2011.

[12] A. Karanasos, J. Ligthart, K. Witberg, G. van Soest, N. Bruining, and E. Regar, "Optical coherence tomography: potential clinical applications," Current Cardiovascular Imaging Reports, vol. 5, no. 4, pp. 206-220, 2012.

[13] L. Meng, B. Lv, S. Zhang, and B. Yv, "In vivo optical coherence tomography of experimental thrombosis in a rabbit carotid model," Heart, vol. 94, no. 6, pp. 777-780, 2008.

[14] A. Phinikaridou, K. J. Hallock, Y. Qiao, and J. A. Hamilton, "A robust rabbit model of human atherosclerosis and atherothrombosis," Journal of Lipid Research, vol. 50, no. 5, pp. 787-797, 2009.

[15] J. Tian, S. Hu, Y. Sun et al., "A novel model of atherosclerosis in rabbits using injury to arterial walls induced by ferric chloride as evaluated by optical coherence tomography as well as intravascular ultrasound and histology," Journal of Biomedicine and Biotechnology, vol. 2012, Article ID 121867, 6 pages, 2012.

[16] Y. Shi, J. E. O’Brien, A. Fard, J. D. Mannion, D. Wang, and A. Zalewski, "Adventitial myofibroblasts contribute to neointimal formation in injured porcine coronary arteries," Circulation, vol. 94, no. 7, pp. 1655-1664, 1996.

[17] M. Zimarino, F. Prati, E. Stabile et al., "Optical coherence tomography accurately identifies intermediate atherosclerotic lesions-An in vivo evaluation in the rabbit carotid artery," Atherosclerosis, vol. 193, no. 1, pp. 94-101, 2007.

[18] H. C. Stary, "Natural history and histological classification of atherosclerotic lesions an update," Arteriosclerosis, Thrombosis, and Vascular Biology, vol. 20, no. 5, pp. 1177-1178, 2000.

[19] H. C. Stary, A. B. Chandler, R. E. Dinsmore et al., "A definition of advanced types of atherosclerotic lesions and a histological classification of atherosclerosis: a report from the Committee 
on Vascular Lesions of the council on arteriosclerosis, American heart association," Circulation, vol. 92, no. 5, pp. 13551374, 1995.

[20] R. Ross and J. A. Glomset, "The pathogenesis of atherosclerosis (first of two parts)," The New England Journal of Medicine, vol. 295, no. 7, pp. 369-377, 1976.

[21] R. Ross and J. A. Glomset, "The pathogenesis of atherosclerosis (second of two parts)," The New England Journal of Medicine, vol. 295, no. 8, pp. 420-425, 1976.

[22] T. Okabe, M. Hoshiga, N. Negoro et al., "Rabbit plaque models closely resembling lesions in human coronary artery disease," International Journal of Cardiology, vol. 147, no. 2, pp. 271277, 2011.

[23] P. Libby, P. M. Ridker, and A. Maseri, "Inflammation and atherosclerosis," Circulation, vol. 105, no. 9, pp. 1135-1143, 2002.

[24] I. K. Jang, G. J. Tearney, B. MacNeill et al., "In vivo characterization of coronary atherosclerotic plaque by use of optical coherence tomography," Circulation, vol. 111, no. 12, pp. 1551-1555, 2005.

[25] T. Yonetsu, T. Kakuta, T. Lee et al., "In vivo critical fibrous cap thickness for rupture-prone coronary plaques assessed by optical coherence tomography," European Heart Journal, vol. 32, no. 10, pp. 1251-1259, 2011.

[26] M. J. Davies, P. D. Richardson, N. Woolf, D. R. Katz, and J. Mann, "Risk of thrombosis in human atherosclerotic plaques: role of extracellular lipid, macrophage, and smooth muscle cell content," British Heart Journal, vol. 69, no. 5, pp. 377-381, 1993.

[27] S. M. Schwartz, R. Virmani, and M. E. Rosenfeld, "The good smooth muscle cells in atherosclerosis," Current Atherosclerosis Reports, vol. 2, no. 5, pp. 422-429, 2000.

[28] P. R. Moreno, K. R. Purushothaman, V. Fuster et al., "Plaque neovascularization is increased in ruptured atherosclerotic lesions of human aorta: implications for plaque vulnerability," Circulation, vol. 110, no. 14, pp. 2032-2038, 2004.

[29] T. Sasaki, M. Kuzuya, K. Nakamura et al., "A simple method of plaque rupture induction in apolipoprotein E-deficient mice," Arteriosclerosis, Thrombosis, and Vascular Biology, vol. 26, no. 6, pp. 1304-1309, 2006.

[30] H. De Leon, J. D. Ollerenshaw, K. K. Griendling, and J. N. Wilcox, "Adventitial cells do not contribute to neointimal mass after balloon angioplasty of the rat common carotid artery," Circulation, vol. 104, no. 14, pp. 1591-1593, 2001. 


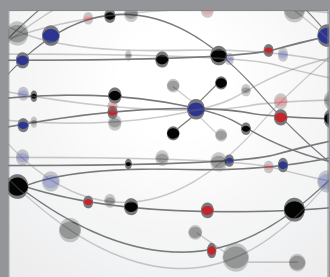

The Scientific World Journal
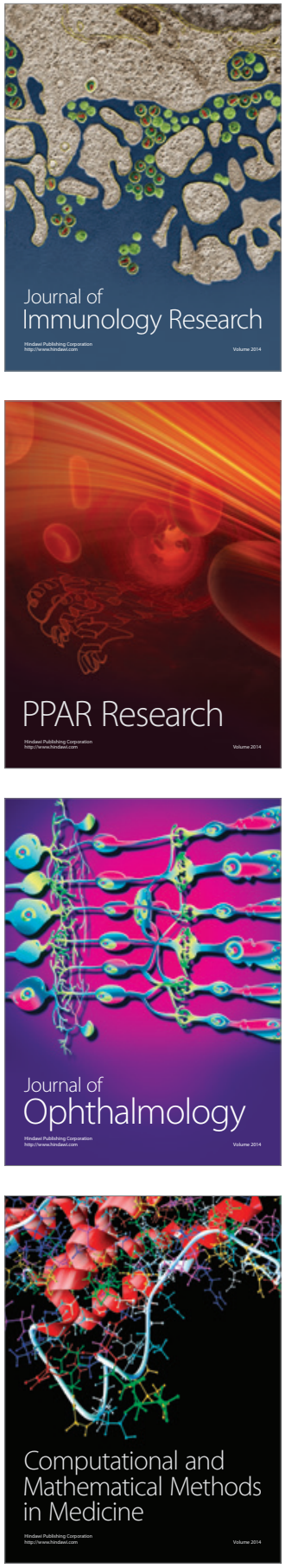

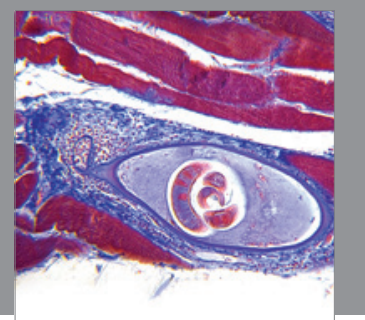

Gastroenterology

Research and Practice
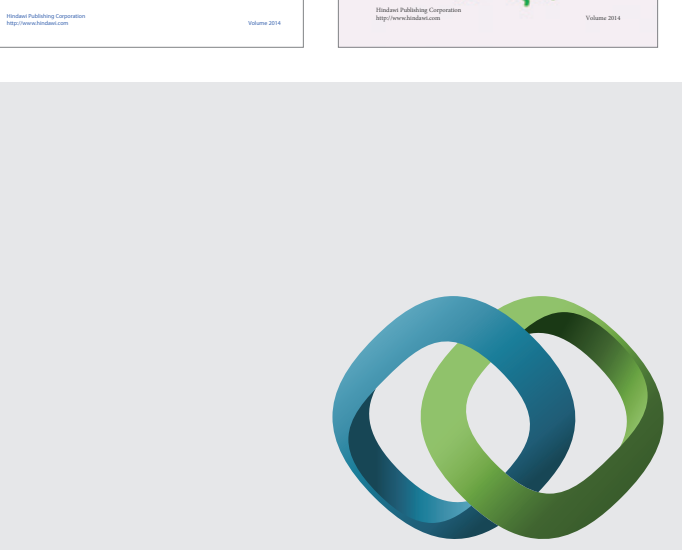

\section{Hindawi}

Submit your manuscripts at

http://www.hindawi.com
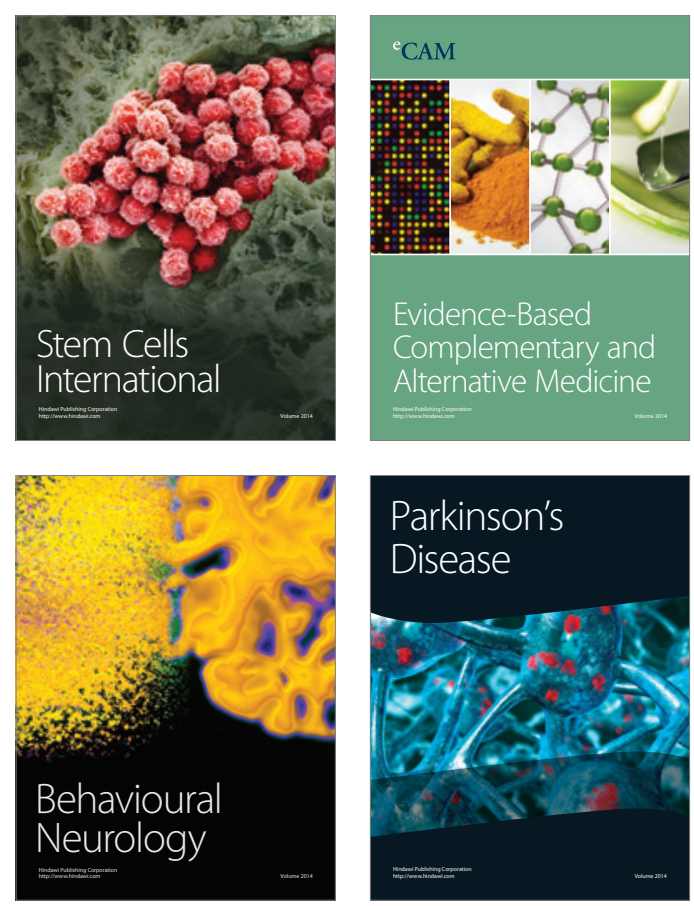

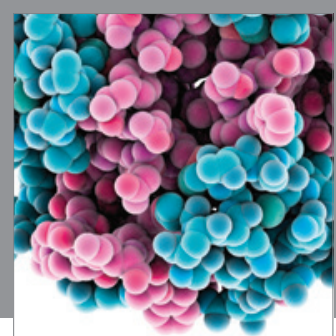

Journal of
Diabetes Research

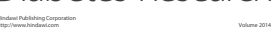

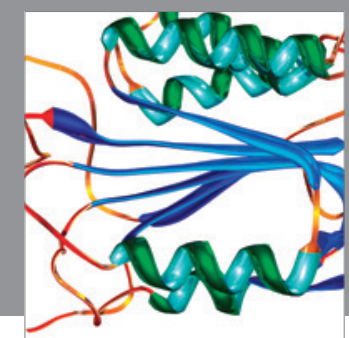

Disease Markers
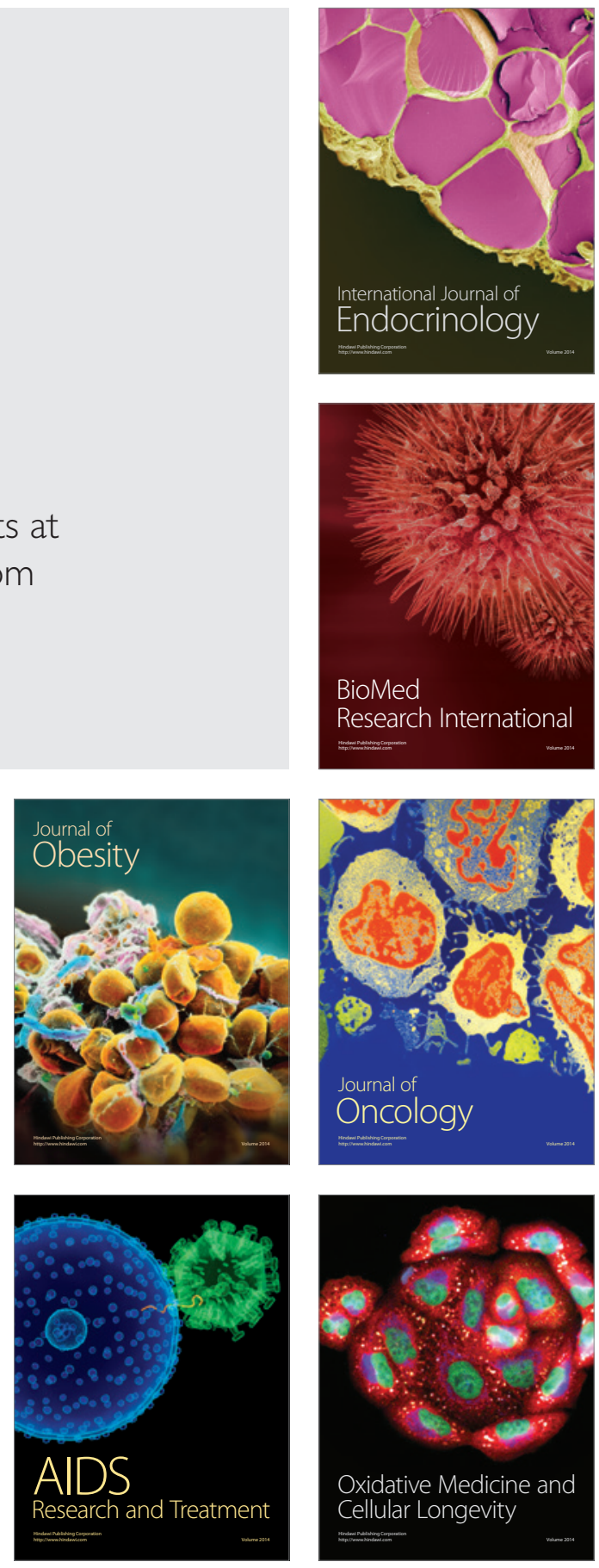